\title{
A IMPLANTAÇÃO DA NORMA NBR 15575 E SEU IMPACTO NO SETOR DE CONSTRUÇÃO CIVIL
}

\author{
THE IMPLEMENTATION OF THE NBR 15575 CODE AND ITS IMPACT ON THE \\ CONSTRUCTION INDUSTRY
}

\author{
(1) Vitor Dias Lopes Nunes ${ }^{1}$ \\ (D) Maria Aparecida Steinherz \\ Hippert ${ }^{2}$

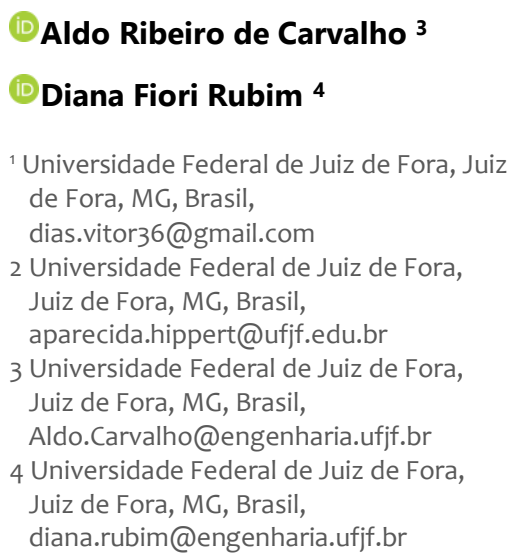

Contribuição dos autores:

VDLN: análise formal, investigação, metodologia, validação, visualização, escrita - rascunho original, escrita - revisão e edição. MASH: conceituação, curadoria de dados, análise formal, aquisição de financiamento, investigação, metodologia, administração do projeto, supervisão, validação, visualização, escrita - rascunho original, escrita - revisão. ARC: investigação, metodologia, visualização, escrita - rascunho original, escrita revisão e edição. DFR: investigação, metodologia, visualização, escrita rascunho original, escrita - revisão e edição.

Fomento: Universidade Federal de Juiz de Fora e Coordenação de Aperfeiçoamento de Pessoal de Nível Superior

Declaração de conflito: nada foi declarado.

Editor(es) Responsável:

Regina C. Ruschel (D)

\begin{abstract}
Resumo
A necessidade de atendimento às exigências dos usuários para um melhor desempenho das edificações tem sido discutida nos últimos tempos, especialmente no Brasil, com a entrada em vigor da norma NBR 15575 - Desempenho de Edificações Habitacionais. A norma estabelece uma série de requisitos a serem atendidos levando a uma mudança na forma como o projeto vem sendo desenvolvido, de maneira geral, no país. O objetivo deste trabalho é levantar e analisar os estudos já realizados referentes à implantação da norma e seu impacto na indústria da construção civil nacional. Como metodologia de pesquisa foi adotada a Revisão Sistemática de Literatura (RSL) de maneira a identificar os estudos de interesse e classificá-los em grupos temáticos segundo os requisitos do usuário estabelecidos pela norma. Os procedimentos metodológicos consistiram basicamente na busca, seleção e avaliação de resultados obtidos em cinco bases de dados nos últimos 10 anos. Cinquenta e um estudos foram selecionados para análise e apontam que, de maneira geral, em que pese os avanços já obtidos alguns obstáculos ainda precisam ser vencidos para uma aplicação mais efetiva da norma. A distribuição dos artigos nos grupos temáticos proporcionou uma visão do atual estágio de desenvolvimento das pesquisas nessa temática podendo ajudar a subsidiar avaliações sobre a natureza das pesquisas realizadas.
\end{abstract}

Palavras-chave: NBR15575. Desempenho. Edificações habitacionais. Norma de desempenho.

\begin{abstract}
The need to meet user requirements to achieve better performance of buildings has been increasingly discussed in recent times, particularly in Brazil, after the release of the NBR 15575 code - Performance of Housing Buildings. This code establishes a series of requirements, inducing a change in the way designs are being developed. This paper aims to identify and evaluate the studies already carried out regarding implementing the NBR 15575 and its impact on the national construction industry. As a research methodology, a Systematic Literature Review was adopted to identify the studies of interest and classify them into thematic groups, according to the user requirements established by the performance standard. The methodological procedure consisted of searching, selecting, and evaluating the five databases' results in the last ten years. Fifty-one studies were selected for analysis. In general, they indicate that, despite the advances already made, some obstacles still have to be overcome in order that the code may be applied more effectively. The articles' distribution into thematic groups provided an overview of the current research stage in this theme and helped to evaluate existing research on the topic.
\end{abstract}

Keywords: NBR 15575. Performance. Housing. Brazilian performance code.

How to cite this article:

NUNES, V. D. L.; HIPPERT, M. A. S.; CARVALHO, A. R. de; RUBIM, D. F. A implantação da norma NBR 15575 e seu impacto no setor de construção civil. PARC Pesquisa em Arquitetura e Construção, Campinas, SP, v. 12, p. e021010, 2021. DOI: http://dx.doi.org/10.20396/parc.v21i00.8656159 


\section{Introdução}

Obras de edificações de qualidade têm sido demandadas pelos diversos intervenientes atuantes no setor da construção civil. A busca pelo desempenho vem de encontro a esta demanda com o estabelecimento de exigências a serem atendidas bem como a forma de mensurá-las. Neste sentido, diferentes países vêm criando normas e estabelecendo legislações e códigos de edifícios voltados para o desempenho, como é o caso do Reino Unido, Nova Zelândia, Austrália, Canadá, Holanda, Suécia, Noruega, Estados Unidos e Espanha (CTE, 2006).

Segundo Oliveira e Mitidieri Filho (2012), em países como França, Canadá e Japão, o desempenho do produto-edifício é primeiro definido para depois serem estabelecidas as tecnologias construtivas a serem adotadas. Esta realidade, porém, ainda não é encontrada no Brasil. No país, o conceito de desempenho, começou a ser discutido na década de 80 quando o Instituto de Pesquisas Tecnológicas do Estado de São Paulo desenvolveu com o apoio do Banco Nacional da Habitação, uma série de requisitos a serem atendidos em projetos habitacionais (BORGES; SABBATINI, 2008).

Outros estudos foram realizados e em 2008 foi publicada a primeira versão da norma brasileira de desempenho, limitada às edificações habitacionais de até cinco pavimentos. A norma passou por discussões dos textos base com toda a cadeia produtiva da construção civil. Em 2013 entrou em vigor oficialmente a versão revisada da norma, com texto mais detalhado e abrangente, direcionada às edificações habitacionais de qualquer porte.

A NBR 15575 (ABNT, 2013), conhecida como Norma de Desempenho (ND) tem o foco nos requisitos dos usuários para o edifício habitacional e seus sistemas, quanto ao seu comportamento em uso, e não na prescrição de como os sistemas são construídos. A norma estabelece a obrigatoriedade de atendimento aos requisitos mínimos de desempenho.

Esta norma é formada por seis partes. A primeira apresenta os requisitos gerais que se aplicam aos edifícios conforme as exigências principais dos usuários. As outras cinco partes são referentes a cada sistema presente em uma edificação: sistemas estruturais, pisos internos e externos, vedações verticais e horizontais, cobertura e sistemas hidrossanitários.

As exigências dos usuários utilizadas para estabelecer os requisitos e critérios presentes na norma são: Segurança (segurança estrutural, segurança contra fogo, segurança no uso e na operação); Habitabilidade (estanqueidade, desempenho térmico, desempenho acústico, desempenho lumínico, saúde, higiene e qualidade do ar, funcionalidade e acessibilidade, conforto tátil e antropodinâmico) e Sustentabilidade (durabilidade, manutenibilidade e impacto ambiental).

Borges e Sabbatini (2008) afirmam ser um grande desafio a tradução das necessidades dos usuários em requisitos e critérios para que possam ser mensurados de maneira objetiva, dentro de determinadas condições de exposição e uso, e que sejam viáveis técnica e economicamente dentro da realidade de cada sociedade, região ou país. Isso porque as exigências do usuário são variáveis, crescentes e subjetivas, o que torna sua definição complexa.

Com o intuito de colaborar com os profissionais da construção civil, algumas instituições e entidades de classe nacionais tem desenvolvido publicações e guias orientativos para ajudar na leitura e entendimento dos requisitos da ND. Este é o caso da Câmara Brasileira da Indústria da Construção - CBIC (CBIC, 2013a; CBIC, 2013b; CBIC, 2015), da Associação 
Brasileira de Escritórios de Arquitetura - ASBEA que junto ao Conselho de Arquitetura e Urbanismo - CAU do Brasil lançou um guia para arquitetos para a aplicação da ND (ASBEA, 2015) e da Associação Brasileira de Engenharia e Consultoria Estrutural - ABECE que publicou uma sugestão para elaboração do memorial descritivo do projeto estrutural (ABECE, 2015).

Ainda como fator impulsionador para a implantação da ND têm-se o Sistema de Avaliação de Conformidade - SiAC do Programa Brasileiro de Qualidade e Produtividade do Habitat - PBQP-H (2017), que estabelece uma série de ações obrigatórias tanto no processo de projeto quanto na obra com a inclusão de procedimentos para a realização de ensaios bem como o controle de aquisição e utilização de materiais considerando o seu desempenho.

A vigência da norma impactou o setor da construção civil de diversas maneiras. As edificações devem atender aos requisitos mínimos estabelecidos, com comprovação a partir de simulações, ensaios de campo e de laboratório. O poder público tem o papel de fiscalizar o atendimento a norma. A construtora/incorporadora tem responsabilidade de garantir condições das obras concluídas. Os fornecedores devem caracterizar e garantir o desempenho dos materiais. E os projetistas devem incorporar o conceito de desempenho nos projetos, especificando materiais, produtos e processos que atendam o desempenho estabelecido pela norma (ABNT, 2013). Com isso a norma gerou a necessidade de alteração no processo de projeto e no planejamento e gestão das empresas.

Desde a sua publicação, estudos têm sido realizados com o intuito de se discutir a implantação da norma, isto é, as facilidades e dificuldades para o atendimento aos requisitos nela estabelecidos bem como os impactos daí decorrentes. Pode-se citar, por exemplo, o trabalho de Silva Junior e Mitidieri Filho (2018) que verificaram a incorporação dos critérios de desempenho em projetos utilizando o Building Information Modeling (BIM). Os autores constataram que cerca de um terço dos critérios da norma são verificáveis através do BIM. Já Pagliari, Costella e Pilz (2018) através de consulta à engenheiros e arquitetos verificaram que os projetistas da região de Chapecó/SC não informam o desempenho e a vida útil, transferindo esta responsabilidade para o construtor que fica dependente dos fornecedores, que conforme o subsistema, ainda não divulgam as informações necessárias.

Neste contexto, o presente trabalho tem por objetivo levantar e analisar dentre os estudos já realizados sobre a Norma de Desempenho, aqueles que tratam da implantação da norma nas empresas e o impacto de sua aplicação na indústria da construção civil nacional.

\section{Metodologia}

Este trabalho utiliza a Revisão Sistemática de Literatura (RSL) como abordagem metodológica. As revisões sistemáticas se apresentam mais abrangentes com questões específicas e protocolos verificáveis e replicáveis por outros pesquisadores (GALVÃO; PEREIRA, 2014). Ela identifica, seleciona e avalia de modo crítico, trabalhos relevantes ao estudo proposto. Isto possibilita o entendimento do contexto no qual a pesquisa está inserida (RANDOLPH, 2009). Para Randolph (2009) a RSL engloba as etapas de planejamento (identificação da necessidade da revisão, definição da questão motivadora da pesquisa, além da elaboração do protocolo de busca), realização (onde ocorre a condução e aplicação dos critérios estabelecidos na fase de planejamento além da síntese e análise dos resultados) e por fim, a comunicação e divulgação dos resultados. 


\section{Planejamento}

A RSL foi proposta com o objetivo de identificar, classificar e analisar os trabalhos referentes à ND de maneira a responder com dados quantitativos e qualitativos à questão motivadora da pesquisa: qual o panorama da produção científica referente à norma NBR 15575 nos últimos dez anos. O levantamento dos estudos primários foi estruturado através do estabelecimento do protocolo de busca, discriminando as estratégias de busca, as bases de dados utilizadas e os critérios de inclusão e exclusão empregados.

Como estratégias de busca, definiu-se que os estudos deveriam estar publicados em dissertações de mestrado e teses de doutorado bem como em periódicos científicos ou anais de congressos disponíveis integralmente em meio eletrônico no intervalo temporal de 2008 a 2018. Esse intervalo de tempo contempla os estudos publicados desde a primeira versão da norma. O termo de busca utilizado foi: "NBR 15575" ou "NBR 15.575" (com aspas). A utilização do numeral com e sem ponto se fez necessária uma vez que em algumas bases, os resultados retornados foram distintos.

A identificação dos estudos foi realizada nas bases de dados: (i) periódicos Coordenação de Aperfeiçoamento de Pessoal de Nível Superior (CAPES), (ii) Catálogo de Teses e Dissertações da CAPES, (iii) Biblioteca Digital Brasileira de Teses e Dissertações (BDTD), (iv) Centro de Referência e Informação em Habitação (InfoHab) e (v) Google Acadêmico. Uma vez que a ND é brasileira, o critério adotado a escolha das bases de dados considerou serem elas nacionais, de grande relevância e amplitude de trabalhos.

Os estudos obtidos foram arquivados e submetidos aos critérios de inclusão e exclusão estabelecidos. Como critérios de exclusão adotaram-se estudos repetidos, que não se encaixavam nos temas considerados e aqueles que não se enquadravam nos tipos dissertações, teses e artigos científicos. Como critérios de inclusão adotaram-se primeiro trabalhos que possuíam, no título NBR 15575 e suas variações (com ponto, sem ponto, com "NBR" ou sem ou, ainda, "Norma de desempenho") ou temas que se relacionassem de modo direto às exigências dos usuários presentes na norma. $E$ segundo, foi realizado a análise dos resumos, que deveria ter relação direta com a NBR 15575.

Os estudos selecionados foram classificados em grupos temáticos segundo as exigências dos usuários presentes na norma. Foi ainda incluído os temas Implementação e impacto e requisitos gerais, que abordam a norma de um contexto mais amplo.

\section{Realização}

A aplicação dos termos de busca foi realizada nos meses de agosto a outubro de $2018 \mathrm{e}$ gerou um total de 1342 publicações (Tabela 1).

Tabela 1 - Número de publicações por base de dados

\begin{tabular}{cc}
\hline Base de dados & Número de publicações \\
\hline Periódicos CAPES & 70 \\
\hline Catálogo CAPES & 154 \\
\hline BDTD & 76 \\
\hline InfoHab & 66 \\
\hline Google & 976 \\
\hline Total & 1342 \\
\hline
\end{tabular}

Fonte: os autores.

Dos resultados obtidos foram eliminados aqueles que não estavam disponíveis. Em seguida foram eliminados os estudos que apareciam em mais de uma base. Seguindo o protocolo estabelecido, foi aplicado o primeiro filtro, com a leitura dos títulos. Em caso 
NUNES, V. D. L.; HIPPERT, M. A. S.; CARVALHO, A. R. de; RUBIM, D. F.

A implantação da norma NBR 15575 e seu impacto no setor de construção civil

de dúvida, os estudos foram mantidos e passou-se à aplicação do segundo filtro, com a leitura dos resumos. Os dados dessas etapas são apresentados na Tabela 2.

Tabela 2: Realização da RSL

\begin{tabular}{cccccc}
\hline Base de dado/etapa RSL & Periódicos CAPES & Catálogo CAPES & InfoHab & $\begin{array}{c}\text { Google } \\
\text { Acadêmico }\end{array}$ & Total \\
\hline Resultados pelo termo de busca & 70 & 154 & 66 & 76 & 976 \\
\hline Estudos disponíveis & 61 & 103 & 62 & 76 & 874 \\
\hline Remoção estudos repetidos & 61 & 100 & 59 & 36 & 798 \\
\hline Filtro 1 -Títulos & 53 & 97 & 53 & 36 \\
\hline Filtro 2 - Resumo & 23 & 90 & 45 & 36 & 1054 \\
\hline
\end{tabular}

Fonte: os autores.

Em seguida os trabalhos foram separados por áreas temáticas. Após a seleção, iniciouse a leitura completa do corpo do texto sendo ainda eliminados aqueles considerados não relevantes para a área temática e para a pesquisa, o que resultou em 292 trabalhos, distribuídos em temáticas segundo a Figura 1. Cabe ressaltar que há estudos que abrangem mais de uma exigência da norma e, portanto, foram considerados em todas elas. Por isso, a diferença entre o somatório de estudos por exigência dos usuários e o número total de estudos identificados.

Figura 1 - Distribuição dos trabalhos identificados na RSL

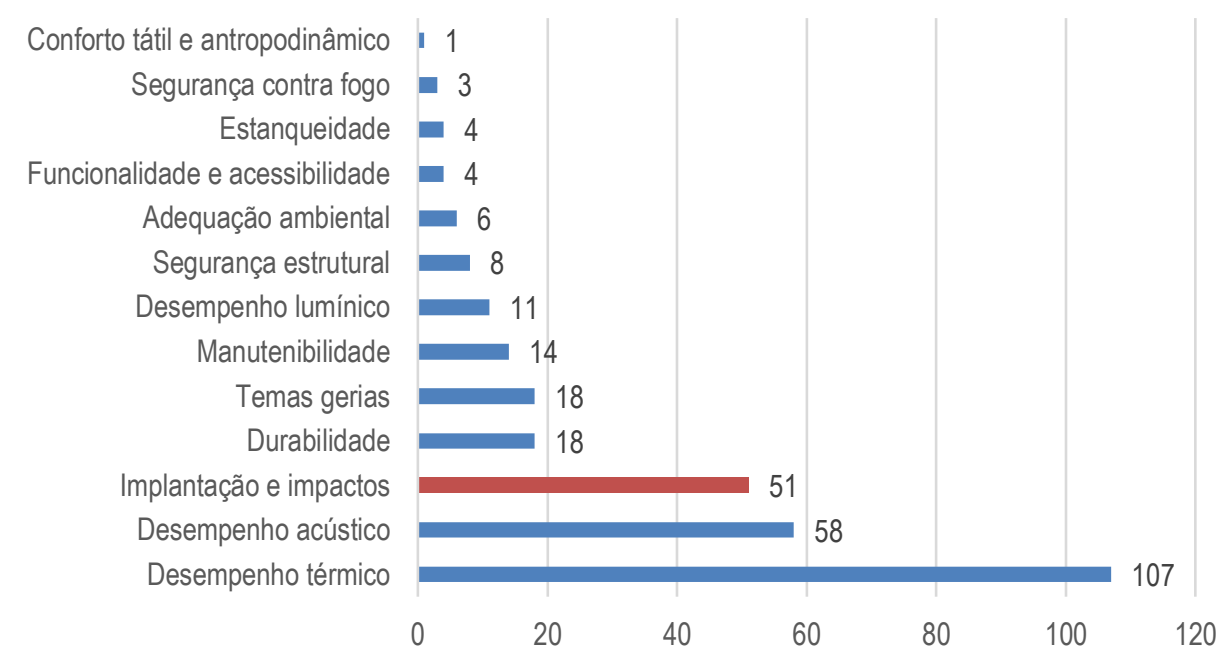

Fonte: os autores.

Para o presente artigo foram selecionados os estudos classificados como implementação e impacto, que relacionam à implantação da norma, suas dificuldades e impactos nos agentes da construção civil, e no processo de projeto. Os 51 trabalhos desta temática são apresentados a seguir e listados no Apêndice $A$.

\section{Resultados sobre a implantação da norma}

Para facilitar o entendimento e análise os resultados foram agrupados em temas: implantação da norma, impactos nas empresas, impactos nos processos de projeto e Recursos de apoio à implantação da Norma.

Com relação à implantação da norma foram identificados estudos relacionados a dois grupos temático: Requisitos e critérios e Intervenientes.

Requisitos e critérios

O desempenho da NBR 15575 é buscado através do estabelecimento de requisitos e critérios, passíveis de serem atendidos e mensurados. Souza (2016) e Souza, Kern e 
Tutikian (2018) analisaram os requisitos da ND e as necessidades para a implantação do nível superior da norma. A NBR 15575:2013 apresenta 155 critérios de avaliação, sendo os requisitos com maior quantidade: desempenho estrutural (29), segurança contra incêndio (23), segurança no uso e na operação (20), durabilidade e manutenibilidade (19) e estanqueidade (17). Segundo os autores, do total de critérios apenas $16 \%$ sofrem variação em função dos níveis de desempenho a serem atendidos (mínimo, intermediário e superior). Porém, a diferença de desempenho entre os níveis inferior e superior é expressiva e pode representar um grande diferencial para a edificação no atendimento a este último.

Mahl e Andrade (2010) observaram, na versão de 2008 da norma, que determinados critérios podem ser facilmente verificados em obra ou através da avaliação de projeto, enquanto outros necessitam de análises mais aprofundadas ou ensaios realizados em laboratório. Entretanto, há uma dificuldade de realização dos ensaios in situ por falta de informação, e os mais complexos por falta de laboratório, além da falta de conhecimento dos profissionais, e custo para a realização dos ensaios.

Ainda com relação aos ensaios, considerando a versão de 2013, Lorenzi (2013) fez uma análise dos ensaios experimentais e evidenciou que existem problemas de interpretação para alguns deles em função da falta de informação e detalhamento, bem como a falta de ensaios que auxiliem no conhecimento do comportamento em uso dos sistemas construtivos das edificações. O autor declara, ainda, que somente ensaios experimentais conseguem avaliar com precisão o desempenho específico das edificações, mas é necessário considerar múltiplas e diversas influências que podem afetar os resultados, se tratando, portanto, de uma tarefa complexa.

Alguns estudos verificaram o atendimento aos requisitos da norma através de estudo de casos em edificações. Um resumo dos mesmos é apresentado no Quadro 1.

\begin{tabular}{|c|c|c|}
\hline Referência & Objeto de estudo & Característica \\
\hline Hybiner et al. (2014) & Condomínio PMCM em Viçosa - MG & $\begin{array}{c}\text { Condomínio vertical } \\
\text { composto por } 5 \text { blocos de } 4 \text { pavimentos }\end{array}$ \\
\hline Lohmann (2015) & $\begin{array}{l}5 \text { condomínios nas cidades de Brusque, Camboriú, Gaspar, } \\
\text { Pomerode e Rodeio na região do Vale do Itajaí - SC }\end{array}$ & $\begin{array}{l}\text { Habitação social térrea pertencente ao Projeto } \\
\text { Reação Habitação }\end{array}$ \\
\hline $\begin{array}{l}\text { Melo, Elias e Fenato } \\
\text { (2016) }\end{array}$ & Residência unifamiliar em Botucatu - SP & Casa alto padrão, com área externa arborizada \\
\hline Costella et al. (2017) & 5 edificações multifamiliares em Chapecó - SC de vários padrões & $\begin{array}{c}1 \text { edificação PMCMV, } 1 \text { baixo padrão, } 1 \text { padrão } \\
\text { médio, } 1 \text { médio alto e } 1 \text { alto. }\end{array}$ \\
\hline
\end{tabular}

Hybiner et al. (2014) constataram manifestações patológicas de diversas naturezas no condomínio estudado, decorrentes tanto de falhas de projeto quanto de execução, além de problemas decorrentes da falta de manutenção, não atendendo aos requisitos mínimos estabelecidos pela norma. Nenhuma das edificações estudadas por Lohmann (2015) atendeu totalmente à ND, sendo alguns critérios atendidos e outros parcialmente. Já a edificação estudada por Melo, Elias e Fenato (2016) atendeu aos critérios de habitabilidade, e durante a concepção do projeto foram realizados todos os estudos demandados, como avaliação das condições naturais do entorno, análise climática, de posição solar, e escolha adequada dos materiais. Costella et al. (2017) declararam que o nível de desempenho das edificações foi abaixo do esperado, com pouca variação de atendimento aos requisitos entre edificações de diferentes padrões, não alçando nem os requisitos mínimos estabelecidos pela norma.

Ainda com relação ao atendimento aos requisitos da norma, Pisani e Zein (2014) realizaram uma pesquisa em dados primários e secundários para avaliar o desempenho de diversos conjuntos habitacionais no Brasil incluídos no Programa Minha Casa Minha 
Vida (PMCMV). Embora tenha sido observada uma grande variação na qualidade dos empreendimentos, nenhum deles atendeu plenamente a ND. Ainda assim, é observado que o Brasil dispõe de conhecimento científico e prática suficiente para a produção de habitação com bom desempenho e qualidade, entretanto ainda faltam ferramentas para que esses parâmetros sejam concretizados em toda a cadeia produtiva.

A necessidade de atendimento dos requisitos da ND pode gerar um aumento dos custos. Segundo Dantas Filho et al. (2014) as mudanças propostas para atendimento aos critérios de desempenho aumentam o custo da construção de Habitação de Interesse Social (HIS) em aproximadamente $12 \%$, sendo o sistema de cobertura o de maior aumento. Nas análises dos autores o aumento de custo foi devido aos materiais que devem ser utilizados para atender os requisitos mínimo da ND, principalmente considerando o conforto térmico e acústico. Tendo por exemplo a adição de mantas nas paredes e na cobertura, ou o emprego de materiais com outras características e maior qualidade. Para os autores este aumento foi considerado alto e pode chegar a inviabilizar os investimentos e financiamentos governamentais.

Buscando o não aumento dos custos, as empresas dos estudos de caso de Lafetá, Fiche e Horta (2017), em Belo Horizonte - MG, e Santos (2018), em Aracajú - SE, não realizavam ensaios nas unidades habitacionais, e em alguns casos nem mesmo realizaram as simulações iniciais requeridas. A não realização dos ensaios estabelecidos pela ND não comprova que a edificação atende às exigências mínimas dos usuários.

Por fim, para avaliar a percepção dos usuários, Prado (2018), realizou entrevistas com moradores de HIS em Sergipe, baseadas nos requisitos da norma e avaliação PósOcupação (APO). Os moradores declararam a qualidade das edificações como positiva, apresentando um índice de satisfação bom para cerca de $74 \%$ dos entrevistados. Entretanto, os empreendimentos, entregues em 2016, ainda não atendem a norma, apresentando inclusive, patologias como umidades, manchas de mofo, trincas e baixo desempenho térmico e acústico. Além disso, foi exposta uma baixa durabilidade de alguns componentes da edificação. As manutenções não eram realizadas de forma adequada, e 54\% dos usuários nunca consultaram o manual de uso, operação e manutenção da edificação. Prado (2018) atribui essa falsa sensação de bom desempenho da edificação a falta de informação dos usuários e a um sentimento de terem realizado o sonho da casa própria, em melhores condições que a anterior.

\section{Intervenientes}

Como a utilização e aplicação do conceito de desempenho exige o envolvimento de diversas áreas de conhecimento, requer também um grande esforço dos agentes da construção civil, especialmente projetistas, construtores, incorporadores, fornecedores, e do poder público.

Para mapear os agentes intervenientes ligados à inspeção predial e compará-los aos intervenientes presentes na ND Bigolin, Pacheco e Silva Filho (2014) utilizaram o método Customer Value Chain Analysis. Observaram que os agentes integrados nos papéis de construtor, incorporador e coordenador de projeto possuem maior importância sobre os demais por serem agregadores de maior número de atividades que geram valor. Esses três papéis representam o centro da cadeia e se caracterizam como principais responsáveis, capazes de realizar a integração e controle dos projetos para garantir o desempenho necessário da edificação. Outros intervenientes como projetistas, fornecedores, laboratórios, poder público, e o usuário final interagem diretamente com o centro da cadeia. 
Aos fornecedores cabe a disponibilização das informações técnicas dos materiais e componentes. Benedicto (2009) após fazer uma análise de dados e um estudo de caso, constatou a existência de divergências entre os fabricantes de materiais hidráulicos e as normas técnicas quanto às suas características e temperatura da água. $\mathrm{O}$ autor sugeriu a revisão das normas, bem como a padronização de ensaios e condições para sua aceitação.

Vale ressaltar que os requisitos da ND são obrigatórios cabendo aos usuários a cobrança pelo seu atendimento. Nicoline (2015) sugere que os municípios passem a incorporar a ND aos códigos de obras e implementem uma política urbana para assegurar o cumprimento das suas funções sociais. Isso porque, Código de Obras é o instrumento legal que estabelece os procedimentos relativos à atividade construtiva, e deve contribuir para a qualidade das obras e do ambiente urbano. $O$ autor afirma que legislação deve, portanto, acompanhar o desenvolvimento e aprimoramento da indústria da construção civil, para garantir a melhoria da qualidade da edificação em todas as suas fases. E que os municípios passem a exigir um maior detalhamento dos projetos arquitetônico e complementares bem como dos seus memoriais descritivos.

No caso de obras públicas, as mesmas estão sujeitas à Lei de Licitações. Ercan (2018) analisou esta lei junto à ND e verificou que não existem requisitos que determinem o nível de desempenho de qualidade da obra e garantam o cumprimento da ND. O autor recomenda a utilização de softwares de gestão para planejamento de obras públicas, uma vez que a garantia da qualidade das edificações tem início na concepção do projeto e vai até o planejamento do processo de execução e manutenção pós-ocupação.

A necessidade de articulação de projetistas, construtores, fornecedores e outros agentes, tais como, instituições e organismos reguladores, em torno da questão de desempenho foi identificada por Castro e Loura (2017). Para os autores, o processo de tomada de decisão deve articular um diálogo entre estes diversos agentes de maneira a contribuir para uma visão mais completa do conceito de desempenho. Entretanto, o questionamento de desempenho ainda é algo em discussão a nível mundial, sendo necessário ser sistematizado. Ainda no processo de tomada de decisão, Otero e Sposto (2016a) geraram um modelo constando aspectos que devem ser considerados prioritários, fundamentada na avaliação dos níveis de risco correlatos e na definição de ações para garantia do desempenho, considerando o equilíbrio entre efetividade e custos destas ações. Assim, o modelo traduz conhecimentos subjetivos em funções matemáticas que podem ser processadas podendo gerar dados concretos para avaliação do desempenho.

\section{Impactos nas empresas}

Alguns autores avaliaram as ações das empresas em função da ND. Para Baldasso (2009), a abordagem de desempenho das edificações é complexa e exige um grande esforço do setor da construção civil para uma maior divulgação e conscientização do uso da norma.

Ao estudarem empresas do ramo da construção civil de Maringá (construtoras e escritórios de arquitetura), Sarvezuk e Silva (2015), observaram que apenas 15\% das empresas buscavam cumprir todas as 6 partes da norma, e apresentavam uma grande dificuldade na fase de adaptação inicial, que acarretaram em diversas alterações nos processos de projeto, como a troca de fornecedores para o atendimento do desempenho mínimo dos materiais, maior qualidade nos detalhamentos e no Manual de Uso e Operação da Edificação, dentre outros. Porém, a tendência é que com o passar do tempo, tais mudanças se incorporassem à rotina das empresas, que superariam os desafios. Por fim, os autores, também verificaram que as principais dificuldades das 
empresas se referem à necessidade de capacitação profissional para o desenvolvimento de projetos que atendam à norma, e um impacto econômico no custo final da obra, uma vez que a prioridade do mercado ainda é o menor custo em detrimento da qualidade. Entretanto, foi não discutido a possível origem do aumento de custos.

Fazendo um levantamento em forma de questionários junto à engenheiros de seis empresas construtoras de grande porte nas cidades de Recife e Olinda, Bento et al. (2016) observaram que os profissionais relataram dificuldade de interpretação da NBR 15575:2013, mas a consideraram vantajosa e importante para a construção civil, aumentando a durabilidade e qualidade da edificação, além de garantir maior conforto ao usuário.

Otero e Sposto (2016b) caracterizaram a atuação das empresas construtoras e incorporadoras de Goiânia frente à ND e observaram que as maiores preocupações consistiam nos requisitos de desempenho acústico, de desempenho térmico e manutenabilidade das edificações. Entretanto, as empresas relataram um maior conhecimento relacionado à segurança estrutural e funcionalidade e acessibilidade.

Para Santos (2018) as empresas vêm dando mais atenção à fase de planejamento e projeto dos empreendimentos, com ênfase na compatibilização de projetos, com o intuito de identificar as falhas antes da execução. Assim, as empresas precisam passar por mudanças a nível organizacional para os setores ligados à parte de planejamento e projeto de empreendimento, tais como: setor de projetos, setor de suprimentos e setor de qualidade. Entretanto, os profissionais entrevistados de Lafetá, Fiche e Horta (2017) relatam aumento dos custos na busca para se adaptar as exigências da ND. Os entrevistados relataram aumento dos custos devido ao emprego de materiais com características mais específicas ao atendimento aos requisitos da norma, e ao controle tecnológico dos ensaios a serem realizados.

A implantação da norma trouxe impactos também à organização interna das empresas construtoras e incorporadoras. A associação entre a ND e os sistemas de qualidade nas empresas foi estudada por Otero e Sposto (2014). Os autores apresentaram uma análise sobre como diferentes processos de um sistema de gestão da qualidade pode auxiliar a empresa no atendimento aos critérios e requisitos da norma. Verificaram que a gestão da qualidade gera diversas ações relacionadas à ND como, por exemplo, controle de projetos, qualificação e contratação de projetistas, laboratórios e fornecedores de materiais e componentes, controle de execução de serviços, avaliação da satisfação de clientes e monitoramento de edifícios pós-ocupação o que pode contribuir para uma utilização mais efetiva da ND.

Santos (2017) também verificou o impacto da aplicação da norma nas empresas de projeto/construção e constatou um cenário ainda de pouca compreensão por parte das empresas sobre a ND. Entretanto, averiguou-se que as empresas maiores, principalmente as de grandes centros, possuem um processo de projeto mais bem definido, o que contribui para adaptação à ND de forma efetiva com a organização dos processos empresariais.

\section{Impactos no processo de projeto}

A partir da análise da norma Gomes (2015) apontou que muitos dos requisitos da NBR 15575:2013 estão relacionados ao projeto. Para verificar como os profissionais estavam lidando com a aplicação da norma em seus projetos, Miranda (2014) avaliou o nível de informação que os arquitetos possuem sobre a norma e as possíveis mudanças advindas na prática da arquitetura. A autora observou que a ND passa a exigir maior conhecimento técnico de materiais e dos sistemas construtivos, além de maior 
conhecimento normativo. Além disso, o processo de projeto passa a ser integrado com todas as disciplinas, com necessidade de definição de materiais e de técnicas construtivas desde as etapas iniciais do projeto de arquitetura, em conformidade com a ND. Essas inter-relações impõem atitudes sistêmicas para as atividades projetuais, que devem resultar em projetos de arquitetura com maior informação gráfica e em memoriais descritivos mais detalhados. Miranda (2014) conclui que a norma exigirá destes profissionais maior domínio e conhecimento sobre os sistemas construtivos inserindo novos procedimentos na rotina projetual, devendo ter mais cuidado na tomada de decisões durante a elaboração e definições dos projetos e da execução. Apesar disso, dentro dos profissionais pesquisados a maioria dos arquitetos não tinha conhecimento sobre a NBR 15575, se estendendo, inclusive, para outras normas técnicas.

Na mesma direção, Okamoto (2015) declara que os profissionais ainda não assimilaram o novo conceito de projetar baseado em desempenho, e que muitas reflexões sobre o tema ainda não foram concretizadas. As adaptações devem ser individuais, personalizadas e dependentes de cada situação e condições de projeto.

O baixo conhecimento da ND foi também verificado por Santos et al. (2017) ao aplicarem vários questionários aos profissionais da região norte do estado do Rio de Janeiro. Cerca de $90 \%$ dos entrevistados não estavam adequando seus projetos à norma mesmo após quatro anos do seu lançamento, menos de 10\% confeccionavam o Manual de Uso Operação e Manutenção das edificações, e apenas 55\% conheciam as consequências de atendimento a normatização brasileira em vigor.

A aplicação dos requisitos de desempenho torna os projetos mais complexos exigindo dos projetistas um maior empenho para as definições de projeto, elaboração das especificações de materiais, memorial descritivo e manual de uso, operação e manutenção (SILVA et al, 2014). Essa exigência foi também verificada por autores como Oliveira e Metidieri Filho (2012), Okamoto (2015), Santos et al. (2016), Santos e Hippert (2016) e Santos (2018) que observaram a necessidade dos projetistas em assumirem uma nova postura, com a aquisição de maior conhecimento técnico das normas a fim de aplicá-lo aos projetos de maneira a garantir o desempenho do produto final.

Santos (2018), além de um melhor detalhamento dos memoriais descritivos e manual dos usuários, identificou a realização de algumas simulações com foco em desempenho térmico, lumínico e acústico. Porém, segundo o autor, não o suficiente para atender aos requisitos da ND e garantir a qualidade do ambiente construído.

Silva et al. (2014) afirmam que é necessário a integração dos profissionais durante o processo de projeto e conhecimento técnico para a busca de novas soluções. Oliveira e Metidieri Filho (2012) recomendam que se faça uma definição dos requisitos no projeto preliminar e busca de tecnologias e soluções disponíveis para atendimento estes requisitos, ponderando custo, benefícios técnicos e prazo.

Para Lorenzi e Silva Filho (2014) a realização de ensaios e verificações nas edificações geram informações que subsidiam a melhoria da qualidade do projeto. Porém, o ideal é que a edificação deseja concebida por desempenho uma vez que a norma pode levar a uma melhoria no processo de projeto, viabilizando a qualificação e aprimoramento dos projetos.

Já Felipe, Roman e Antunes (2016), ao realizarem a análise de projetos e memoriais, constataram a eficácia da norma em evidenciar itens que obrigatoriamente deveriam estar nos projetos. Os autores afirmam ainda que apenas pela verificação em projeto é possível prever possíveis falhas de desempenho nas edificações, do mesmo modo a 
inexistência de alguns detalhamentos e a falta de compatibilização entre os diversos projetos da edificação, tenderá ao não atendimento aos requisitos mínimos propostos pela ND.

Cotta (2017) e Cotta e Andery (2018) observaram alguns desafios a serem enfrentados pelas empresas e também identificaram falta de conhecimento a respeito da norma e das exigências relacionadas ao processo de projeto. Neste sentido, propuseram ferramentas e procedimentos para a gestão do processo de projeto de maneira a garantir o desempenho. Os autores desenvolveram um fluxo de atividades para o processo de projeto e ferramentas de suporte técnico e gerencial para facilitar a implantação da ND. As ferramentas utilizadas basearam-se no conceito de engenharia simultânea para proporcionar a integração entre processos, disciplinas e funções, estimulando a colaboração entre os agentes, de forma a diminuir a falta de conhecimento dentro das construtoras e nas empresas de projeto sobre as questões técnicas e gerenciais, a fim de garantir o desempenho do produto final.

Já Gomes (2015), em seu estudo de caso, propõe soluções construtivas, que podem ser indicadas no projeto objetivando o atendimento a ND, focando nos detalhes relacionados ao desempenho térmico e desempenho acústico dos sistemas de vedações verticais, horizontais e sistemas de cobertura, podendo inclusive atingir o nível superior de desempenho.

Com relação aos projetos de sistemas prediais, Cover (2012) elaborou diretrizes baseadas na ND a serem utilizadas em licitações de projetos hidrossanitários. A utilização de diretrizes para a fundamentação do projeto colabora com o conceito de desempenho, auxiliando os projetistas a projetarem dentro dos parâmetros propostos. O autor declara que deve ser realizada uma gestão da manutenção dos sistemas, e o projeto deve buscar a redução do índice de manutenção corretiva e aumento da vida útil.

Miyazato (2012) buscou identificar maneiras de integrar um sistema de aquecimento solar de água a uma edificação para facilitar o atendimento à ND e a partir daí também gerou diretrizes de projetos priorizando as necessidades dos usuários. A pesquisa mostrou que parte dos projetistas não estão capacitados para executar as atividades necessárias.

Ainda com relação aos projetos de sistemas prediais, Pinheiro (2017) ao abordar o processo de projeto verificou que existem poucos estudos sobre a temática na literatura. A autora realizou entrevistas junto a empresas do setor (escritórios de arquitetura, empresas construtoras e escritórios de projetos de sistemas prediais) e verificou que passados três anos (à época do trabalho) da entrada em vigor da norma ainda existiam profissionais que a desconheciam. Além disso, observou que ainda existe uma desvalorização do projeto, sendo retratado tardiamente, separadamente e sem colaboração e integração entre os profissionais. Porém, segundo a autora as responsabilidades impostas pela norma têm impulsionado, embora de forma lenta, uma maior aproximação de projetistas e empreendedores através dos conceitos da Engenharia Simultânea.

Lima (2016) e Lima, Andery e Viega (2016) observaram que as empresas e projetistas, normalmente, não têm preocupações com o projeto hidrossanitários e são encontradas dificuldades para obtenção de dados técnicos e realização de ensaios específicos associados aos requisitos normativos. Os principais problemas, além do conhecimento técnico, consistem no envolvimento tardio dos projetistas na concepção do projeto e de sua participação descontinuada e pontual, com falta de interação entre os profissionais, e deixando de incluir o desenvolvimento do as built e avaliações pós- 
ocupação. A integração entre as etapas de projeto e execução deve contar com colaboração da equipe de obra já na fase de concepção, bem como do projetista na etapa de execução dos empreendimentos, realizando o acompanhamento e vistoria das instalações. Os autores observaram uma gradativa valorização da atividade projetual, principalmente em situações que as empresas se preocupam com redução dos riscos, o que implica na necessária valorização das disciplinas de engenharia no processo de projeto.

\section{Recursos de apoio à implantação da Norma}

De maneira a subsidiar o trabalho dos profissionais envolvidos na aplicação da ND, Oliveira et al. (2013) geraram uma lista sistematizando o conjunto de normas que complementam a NBR 15575:2013, de modo a identifica-las sem que haja necessidade de uma consulta minuciosa a todas as partes da ND.

Caiado (2014) elaborou uma tabela com avaliação dos materiais segundo a ND e selos sustentáveis, de modo a colaborar com os fabricantes que precisam indicar as propriedades dos produtos, comprovando a sua conformidade, e apresentar os resultados de forma clara e transparente, e aos projetistas que necessitam especificar os produtos de acordo com as suas propriedades para atender às necessidades do projeto.

Moura (2015) gerou um catálogo com os produtos inovadores que atendem a norma, baseado nas informações divulgadas na Revista Techne entre os anos de 2005 a 2015. O autor identificou 164 inovações nas diversas áreas, que foram classificados de acordo com sua aplicabilidade baseada na ND. O autor destaca, ainda, que o reduzido número de fornecedores e o alto custo de aquisição, que envolve tanto a antecipação da compra de materiais como a necessidade de uso de equipamentos de grande porte durante o processo de construção, dificulta a inserção de algumas inovações no mercado da construção civil.

Carvalho (2017) gerou uma lista de documentos técnicos a serem solicitados em um procedimento de vistoria de recebimento de uma edificação habitacional a partir dos parâmetros estabelecidos pela ND e utilizando a ferramenta de gestão da qualidade $5 \mathrm{~W} 1 \mathrm{H}$. A sistematização dos documentos se torna útil em um procedimento de vistoria de recebimento de uma edificação habitacional, uma vez que subsidia os profissionais de engenharia ou arquitetura - representando os interesses dos futuros usuários do empreendimento - nos procedimentos formais de recebimento de obras a serem entregues pela empresa responsável por sua construção.

O uso dos recursos de Tecnologia tem sido crescente nas diversas áreas do setor de construção civil. Neste cenário é crescente o potencial de utilização do BIM. Coelho (2013) apresentou o uso do Revit para algumas verificações de desempenho estabelecidas na ND. A autora conclui que o software tem condições de realizar simulações simplificadas que auxiliam na concepção do projeto. Silva Jr. (2016) propôs a incorporação do BIM, através do Revit, no desenvolvimento de projeto de arquitetura para atendimento aos requisitos da Norma e concluiu que $1 / 3$ dos requisitos podem ser verificados de maneira automática. Silva (2017) e Silva e Arantes (2017) também estudaram a aplicabilidade de verificação dos requisitos de projeto da ND, utilizando o software Solibri Model Checker (SMC). Os autores também constataram a possibilidade de checagem de $1 / 3$ dos requisitos da Norma. Além disto, verificaram uma redução de $60 \%$ do tempo de conferência de projetos além da identificação de $22 \%$ a mais de não conformidades. 


\section{Discussão}

A implantação da ND demanda o atendimento aos requisitos nela contidos. Nos estudos analisados são apontadas algumas dificuldades para o atendimento a estes requisitos. Uma primeira dificuldade refere-se à realização de alguns dos ensaios presentes na norma. É o caso, por exemplo, dos ensaios de ação de calor e choque térmico para sistema de vedação vertical externo (SVVE), impacto de corpo mole em SVVE e ensaio de desempenho térmico por medição em campo (LORENZI, 2013). Além disso, a necessidade de realização de ensaios mais complexos, que dependem de laboratórios, traz dificuldades, tanto pelo custo dos ensaios, quanto pela falta de laboratórios em determinadas regiões (MAHL; ANDRADE,2010; LAFETÁ, FICHE; HORTA,2017; SANTOS,2018). O CBIC (2013a) levantou a capacitação técnica e laboratorial relativa à ND e encontrou somente 20 instituições que se distribuem na realização dos ensaios requeridos.

Outra dificuldade apontada refere-se ao aumento de custos específicos para atendimento aos requisitos da norma (MAHL; ANDRADE, 2010; DANTAS FILHO ET. AL, 2014; SARVEZUK; SILVA, 2015; LAFETÁ; FICHE; HORTA, 2017). Os profissionais relataram, a necessidade de utilização de materiais de maior de qualidade ou adição de elementos, como por exemplos mantas nas paredes e lajes, para atingir os requisitos de desempenho térmico e acústico. Gerando um aumento de custo em relação ao produto anteriormente executado. Além disso, ainda há no mercado uma preferência por custo inicial menor, contrapondo a qualidade do ambiente construído e o custo global da edificação.

Esta, porém não é a realidade encontrada pelas grandes empresas, que possuindo uma melhor organização, e um sistema de gestão de qualidade (OTERO; SPOSTO, 2014; SANTOS, 2017), considerando que já contratavam seus projetos junto às empresas especializadas e suas edificações eram construídas atendendo às normas prescritivas presentes na ND.

Além disto, de maneira geral, os trabalhos indicaram o não atendimento aos requisitos de desempenho da norma e consequentemente às necessidades dos usuários (HYBINER et al., 2014; PISANI; ZEIN, 2014; LOHMAN, 2015; COSTELLA et al., 2017; PRADO, 2018). Entretanto, cabe ressaltar que a NBR 15575 passou a ser compulsória para as obras protocoladas nas prefeituras a partir de julho de 2013. Todas as obras protocoladas anteriormente, independentemente do momento de sua entrega, não são obrigadas a cumprir a NBR 15575.

O usuário leigo, não tem conhecimento para fiscalizar e cobrar o desempenho das edificações, e como constatado por Prado (2018) em casos relacionados ao PMCMV há um sentimento de satisfação associado sonho da "casa própria". Cabe, portanto, destacar o papel dos órgãos públicos na cobrança pelo atendimento à ND, segundo instrumentos já existentes, que poderiam ser revistos. Por exemplo, a nível municipal, a norma poderia ser incorporada aos códigos de obras, conforme proposto por Nicoline (2015). A verificação dos requisitos em projeto, seriam realizadas antes da liberação da "licença para construção", e a comprovação da realização dos ensaios seria utilizada para a liberação do "auto de conclusão de obra" (Habite-se).

No caso de obras públicas, a lei de licitações a que estão sujeitas, não contempla requisitos de desempenho, conforme verificado por Ercan (2018). O PBQP-H incluiu, em 2017, no SiAC, a obrigatoriedade do atendimento à NBR 15575. Fortalecendo a necessidade de cumprimento dos seus requisitos, uma vez que o PBQP-H é uma certificação exigida pela Caixa Econômica (e outras instituições financeiras) para obtenção de financiamentos e para participação de licitações públicas. 
Ao verificarem o impacto da implantação da norma nas empresas, os autores, observaram que as empresas também relataram dificuldades para se adaptarem à ND. Isso devido à necessidade de mudanças, associada à falta de informação técnica, levando a necessidade de capacitação dos profissionais (SARVEZUK; SILVA, 2015; BENTO et al., 2016; SANTOS, 2017).

Foi identificada, também, a necessidade de mudança a nível organizacional nos setores de planejamento e projeto, com intuito de reduzir problemas em obras (Santos, 2018). Porém, para Santos (2017) este cenário de dificuldades difere em empresas maiores que já possuem um processo de projeto mais definido, o que contribui para a melhor organização de seus processos empresariais. Otero e Sposto (2014) verificaram, ainda, que um sistema de gestão de qualidade pode auxiliar as empresas a se adaptarem mais facilmente à ND uma vez que elas estão mais estruturadas possuindo melhor organização e aplicação de ferramentas para planejamento, execução, monitoramento e controle das ações.

Quanto ao projeto, Gomes (2015) verificou que muitos dos requisitos da norma estão relacionados ao projeto. Deste modo, para o atendimento à ND os projetistas devem repensar o projetar, e a edificação deve ser concebida a partir do conceito de desempenho. E com isso, o processo de projeto, no geral, se tornou mais complexo (SILVA et al, 2014; OLIVEIRA; METIDIERI FILHO, 2012; OKAMOTO, 2015; SANTOS et al., 2016; SANTOS; HIPPERT, 2016; SANTOS, 2018).

Neste contexto os autores destacam a necessidade de integração dos profissionais ao longo do processo de projeto (SILVA et al., 2014; COTTA, 2017; PINHEIRO, 2017; COTTA; ANDERY, 2018) além de reforçar a necessidade de valorização do projeto para atendimento aos requisitos normativos (LIMA, 2016; LIMA; ANDERY; VIEGA, 2016). Esta integração deve considerar os intervenientes, isto é, incorporadores, projetistas, construtores e fornecedores com a definição de materiais e sistemas construtivos desde a fase inicial do projeto. A integração entre as disciplinas do projeto pode ser obtida com a adoção da Engenharia Simultânea, que substitui as tentativas de compatibilização das etapas concluídas de forma individual pelos projetos. Isto pode ser obtido com o uso do BIM, que ao criar um modelo único do projeto é capaz de facilitar a interoperabilidade entre projetos e mesmo na integração entre os profissionais de várias disciplinas.

A ND apresenta uma grande quantidade de informação e o tema de desempenho é considerado complexo. Neste sentido, alguns estudos apresentam propostas para auxiliar os profissionais na realização de suas tarefas. É o caso da listagem de normas constantes na ND (OLIVEIRA et al., 2013), da tabela com propriedade dos materiais (CAIADO, 2014), catálogo de produtos inovadores (MOURA, 2015) bem como a listagem de documentos técnicos necessários para recebimento de uma edificação (CARVALHO, 2017) e ainda as ferramentas de gestão para auxiliar na implantação da norma (COTTA, 2017; COTTA; ANDERY, 2018)

O uso de softwares se destaca neste contexto ajudando na verificação dos requisitos de desempenho. A utilização do BIM demonstra grande potencial para auxiliar o atendimento aos requisitos de desempenho da ND. É possível a verificação de requisitos durante a concepção do projeto, e maior detalhamento dos produtos e materiais utilizados. Além disso, o BIM também é uma ferramenta capaz de facilitar a interoperabilidade entre projetos e na integração entre os profissionais de várias disciplinas (COELHO, 2013; SILVA JR., 2016). Silva (2017) e Silva e Arantes (2017) ainda demonstram que o software SMC pode verificar grande parte dos requisitos da ND.

Essa complexidade dos projetos tem demandado um maior conhecimento técnico dos profissionais. A falta de conhecimento dos profissionais para lidar neste contexto foi 
apontada por autores como Miranda (2014), Okamoto (2015), Santos (2017), Cotta (2017) e Cotta e Andery (2018) relacionados aos projetos de arquitetura, bem como por Miyazato (2012) Lima (2016), Lima, Andery e Viega (2016) e Pinheiro (2017), para os projetos de instalações.

Em que pese as ações que vêm sendo realizadas pelas instituições e entidades de classe, há o desenvolvimento de publicações e guias orientativos, conforme visto anteriormente. Entretanto, dado a falta de conhecimento à NBR 15575 dos profissionais brasileiros em atuação, o CREA e o CAU devem atuar mais enfaticamente, com o desenvolvimento de ações para adaptação dos profissionais já habilitados. Podendo utilizar práticas que foram adotas em outros países, como a Espanha, por exemplo, quando da implantação de sua ND, estabelecendo etapas e provendo cursos, palestras, eventos, ambientes de interação e disponibilizando livre acesso a conteúdo para a adequação técnica dos profissionais, além de incentivo do governo para adaptação das empresas (KERN; SILVA; KAZMIERCZAK, 2014). Assim, adequando engenheiros e arquitetos atuantes às necessidades de desempenho das edificações requeridas pelos usuários.

Mas mais do que isso, é preciso que o conceito de desempenho seja discutido ainda durante a formação dos profissionais, sejam eles arquitetos ou engenheiros, para que cheguem ao mercado com a capacidade técnica necessária para atender às exigências dos usuários.

\section{Considerações finais}

O tema desempenho de edificações tem crescido de importância, especialmente no Brasil, desde a publicação da primeira versão da NBR 15575 - Norma de Desempenho, em 2008. Neste contexto, este artigo teve por objetivo identificar e analisar os estudos existentes em dissertações, teses e artigos científicos entre 2008 e 2018, que tratam da NBR 15575, a partir de uma RSL realizada nas bases de dados do periódico CAPES, catálogo de Teses e Dissertações da CAPES, Biblioteca Digital Brasileira de Teses e Dissertações, InfoHab e Google Acadêmico. Os estudos identificados foram classificados por área temáticas. Neste artigo foram analisados 51 estudos que consideram a implantação da norma e seu impacto no setor da construção civil.

Nos estudos que analisaram os requisitos da ND, verificou-se a falta de laboratórios para a realização dos ensaios demandados. Já os estudos de casos realizados indicaram que as edificações ainda não estão atendendo à ND.

Os estudos, também, abordaram os intervenientes atuantes no processo construtivo apresentados na norma. O papel de incorporador/construtor/coordenador se apresenta como o centro da cadeia e da gestão das informações. A disponibilização de informações dos produtos por seus fornecedores ainda não é prática comum, o que acaba por dificultar a tarefa de especificação dos materiais pelos projetistas. A estes cabem o atendimento da maior parte dos requisitos da norma. Os projetos tornam-se mais complexos exigindo melhores definições de projeto e de especificações. Por outro lado, a existência de Sistemas de Qualidade na empresa pode funcionar como um elemento facilitador para a implantação da ND tem em vista, por exemplo, as exigências do PBQP-H.

Como o processo de projeto se tornou mais complexo, a Integração entre processos, disciplinas e funções, associada a colaboração entre os agentes, fomenta o conhecimento dentro das empresas, e contribui para o desenvolvimento de edificações de qualidade que satisfaçam as exigências dos usuários. 
Do exposto vê-se que apesar do tempo decorrido e da divulgação realizada, o desconhecimento da norma ainda se faz presente, demandando a realização de ações para um maior entendimento da necessidade de atendimento bem como da implantação dos seus requisitos. A NBR 15575 embora não seja lei tem força de lei, segundo o Código do Consumidor, cabendo ao usuário o papel de fiscalização do seu cumprimento.

\section{Agradecimentos}

À Universidade Federal de Juiz de Fora pela bolsa de iniciação científica (Projeto 45047) e Coordenação de Aperfeiçoamento de Pessoal de Nível Superior (CAPES) pela bolsa de mestrado junto ao Programa de Demanda Social no Programa de Pós-graduação em Ambiente Construído.

\section{Referências}

ABECE - ASSOCIAÇÃO BRASILEIRA DE ENGENHARIA E CONSULTORIA ESTRUTURAL. Memorial descritivo do projeto estrutural (de edifícios residenciais em concreto armado). São Paulo, 2015.

ABNT - ASSOCIAÇÃO BRASILEIRA DE NORMAS TÉCNICAS. NBR 15575: Edificações Habitacionais - Desempenho. Rio de Janeiro, 2013.

ASBEA - ASSOCIAÇÃO BRASILEIRA DE ESCRITÓRIOS DE ARQUITETURA. Guia para arquitetos na aplicação da norma de desempenho ABNT NBR 15.575. São Paulo, 2015.

BORGES, C. A. de M.; SABBATINI, F. H. O Conceito de desempenho de edificações e a sua importância para o setor da construção civil no Brasil. Boletim Técnico da Escola Politécnica da USP, 2008.

CBIC - CÂMARA BRASILEIRA DA INDÚSTRIA DA CONSTRUÇÃO. Desempenho de edificações habitacionais: guia orientativo para atendimento à norma ABNT NBR 15575/2013. Brasília. 2013 a.

CBIC - CÂMARA BRASILEIRA DA INDÚSTRIA DA CONSTRUÇÃO. Dúvidas sobre a norma de desempenho: especialistas respondem às principais dúvidas e elencam requisitos de suporte para elaboração de projetos. Brasília. 2015.

CBIC - CÂMARA BRASILEIRA DA INDÚSTRIA DA CONSTRUÇÃO. Manual de uso, operação e manutenção das edificações: orientações para construtoras e incorporadoras. Brasília. 2013b.

CTE - CÓDIGO TÉCNICO DE LA EDIFICACIÓN. Madrid, Gobierno Espanhol. Madrid. 2006. Disponível em: http://www.codigotecnico.org. Acesso em: 01 jul. de 2017

GALVÃO, T. F.; PEREIRA, M. G. Revisões sistemáticas da literatura: passos para sua elaboração. Epidemiol. Serv. Saúde, Brasília, DF. v.23, n.1, p.183-184, jan./mar. 2014.

OLIVEIRA, L. A.; MITIDIERI FILHO, C. V. O PROJETO DE EDIFÍCIOS HABITACIONAIS CONSIDERANDO A NORMA BRASILEIRA DE DESEMPENHO: ANÁLISE APLICADA PARA AS VEDAÇÕES VERTICAIS. Gestão \& Tecnologia de Projetos, [S. I.], v. 7, n. 1, p. 90-100, 2012. DOI: https://doi.org/10.4237/gtp.v111.208.

PBQP-H - PROGRAMA BRASILEIRO DE QUALIDADE E PRODUTIVIDADE DO HABITAT. Sistema de Avaliação de Conformidade - SiAC. 2017. Disponível em: <http://www.cidades.gov.br/pbqp-h>. Acesso: 03 jun. 2017.

RANDOLPH, J. J. A guide to writing the dissertation literature review. Practical Assessment, Research \& Evaluation, V. 14, n. 11, p. 1-13, jun. 2009.

SILVA JUNIOR, M. A.; MITIDIERI FILHO, C. V. Verificação de critérios de desempenho em projetos de arquitetura com a modelagem BIM. PARC Pesquisa em Arquitetura e Construção, v. 9, n. 4, p. 334-343, 1 dez. 2018. DOI: https://doi.org/10.20396/parc.vgi4.8650453. 
NUNES, V. D. L.; HIPPERT, M. A. S.; CARVALHO, A. R. de; RUBIM, D. F.

A implantação da norma NBR 15575 e seu impacto no setor de construção civil

\section{Apêndice A: Artigos classificados pela RSL}

BALDASSO, P. C. P. A norma de desempenho de edificações e seu impacto na cadeia produtiva da construção civil brasileira. In: CONFERÊNCIA INTERNACIONAL DA LARES, 9., 2009, São Paulo. Anais [...]. São Paulo: LARES, 2009.

BENEDICTO, Sérgio Murilo de Oliveira. Desempenho de sistema predial de água quente. 2009. Dissertação (Mestrado em Construção Civil) - Programa de Pós-Graduação em Construção Civil, Universidade Federal de São Carlos, São Carlos, 2009.

BENTO, A.; NEVES, D.; PIRES, J.; OLIVEIRA, M.; SILVA; D. A influência da NBR 15575 (2013) na durabilidade e vida útil das edificações residenciais. In: SEMINÁRIO DE PATOLOGIA E RECUPERAÇÃO ESTRUTURAL, 1., 2016, Recife. Anais [...]. Recife: Universidade de Pernambuco, 2016.

BIGOLIN, M.; PACHECO, L.S.; SILVA FILHO, L.C.P. Inspeção Predial e Norma de Desempenho: Agentes Intervenientes. In: CONGRESSO BRASILEIRO DO CONCRETO, 56., 2014, Natal. Anais [...]. Natal: IBRACON, 2014.

CAIADO, Alessandra Ramos. Contribuição ao estudo da rotulagem ambiental dos materiais de construção civil. 2014. Dissertação (Mestrado em Arquitetura e Urbanismo) - Faculdade de Arquitetura e Urbanismo, Universidade de São Paulo, São Paulo, 2014.

CARVALHO, Letícia Moura de. Proposta para recebimento de obras conforme os requisitos da NBR 15575 usando ferramentas da qualidade. 2017 Dissertação (Mestrado em Engenharia Industrial) - Programa de Pós-graduação em Engenharia Industrial, Escola Politécnica, Universidade Federal da Bahia, Salvador, 2017.

CASTRO, M. L. A. C. DE; LOURA, R. M. Requisitos e critérios para projetos habitacionais: em busca de um desempenho ampliado. Paranoá: cadernos de arquitetura e urbanismo, v. 19, n. 19, 3 mar. 2018. DOI: https://doi.org/10.18830/issn.1679-0944.n19.2017.09

COELHO, Fernanda Fonseca de Melo. O potencial e limitações do BIM - Building Information Modeling para o atendimento da ABNT NBR 15575. 2013. Dissertação (Mestrado em Concentração: Projeto, Produção e Gestão do edifício) - Programa de Pós-Graduação em Arquitetura e Urbanismo, Universidade Federal Fluminense, Niterói, 2013.

COSTELLA, M. F.; CARUBIM, K.; PAGLIARI C. S.; SOUZA, N. S. DE. Avaliação da aplicação da norma de desempenho: estudo de caso em cinco empreendimentos. Revista de Engenharia Civil IMED, Passo Fundo, vol. 4, n. 2, p. 55-74, Jul./Dez. 2017

COTTA, A. C.; ANDERY, P. R. P. As Alterações no Processo de Projeto das Empresas Construtoras e Incorporadoras Devido à NBR 15575 - Norma de Desempenho. Ambiente Construído, Porto Alegre, v. 18, n. 1, p. 133-152, jan./mar. 2018. DOI: https://doi.org/10.1590/s1678-86212018000100213.

COTTA, Ana Cláudia. Contribuição ao estudo dos impactos da NBR 15575:2013 no processo de gestão de projetos em empresas construtoras de pequeno e médio porte. 2017. Dissertação (Mestrado em Construção Civil) Programa de Pós-Graduação em Construção Civil, Escola de Engenharia, Universidade Federal de Minas Gerais, Belo Horizonte, 2017.

COVER, Marcelo Pinarelli. Diretrizes para licitação de projetos de sistemas prediais de água fria e esgoto sanitário em campi universitários. 2012. Dissertação (Mestrado em Construção Civil) - Programa de Pós-Graduação em Estruturas e Construção Civil, Universidade Federal de São Carlos, São Carlos, 2012.

DANTAS FILHO, J. B. P.; GUEDES, J. P.; CÂNDIDO, L. F.; BERTINI, A. A.; NETO, J. L. T. The costs of the performance standards in a social housing using the BIM platform. In: IAHS WORLD CONGRESS ON HOUSING, 40., 2014, Funchal. Proceedings [...]. Funchal: IAHS, 2014.

ERCAN, Luana Maris Pedrosa Cruz. Diretrizes e requisitos para o planejamento de obras públicas a partir da análise de processos licitatórios. 2018 Dissertação (Mestrado em Construção Civil) - Programa de Pós-Graduação em Construção Civil, Escola de Engenharia, Universidade Federal de Minas Gerais, Belo Horizonte, 2018. 
FELIPE F. M.; ROMAN, H. R.; ANTUNES, E. G. P. Análise do auxílio dos métodos de avaliação e critérios de desempenho da ABNT NBR 15.575:2013 no processo do diagnóstico das fissuras - Estudo de Caso. In: CONGRESSO BRASILEIRO DE PATOLOGIA DA CONSTRUÇÃO, 2., 2016. Belém do Pará. Anais [...]. Belém: CBAT, 2016.

GOMES, João Eduardo Veloso. Avaliação do desempenho de edifícios segundo a norma NBR 15575 adaptação ao caso de edifício reabilitado. 2015. Dissertação (Mestrado em Engenharia) - Departamento de Engenharia Civil, Faculdade de Engenharia da Universidade do Porto, Porto, Portugal, 2015

HYBINER, J. M. B. M.; TIBIRIÇÁ, A. C. G.; CARVALHO, A. W. B.; MURAT, M. G.; HOSKEN, C. uso da NBR 15575: 2013 na avaliação técnico-construtiva de um conjunto habitacional. ENCONTRO NACIONAL DE TECNOLOGIA DO AMBIENTE CONSTRUÍDO, 15., 2014, Maceió. Anais [...]. Porto Alegre: ANTAC, 2014.

KERN, A. P.; SILVA, A.; KAZMIERCZAK, C. S. O processo de implantação de normas de desempenho na construção: um comparativo entre a Espanha (CTE) e Brasil (NBR 15575/2013). Gestão e Tecnologia de Projetos, São Paulo, v. 9 , n. 1, p. 89-101, jan./jun. 2014. DOI: https://doi.org/10.11606/gtp.vgi1.89989

LAFETÁ, B. D. A.; FICHE, L. R.; HORTA, F. S. Avaliação das mudanças das diretrizes acústicas após a revisão da NBR 15575 (2013) em Belo Horizonte. Revista Petra, Belo Horizonte, v. 3, n. 2, p. 163-178, ago./dez. 2017.

LIMA, C. F. M.; ANDERY, P. R. P.; VEIGA, A. C. R. Análise do processo de projeto de sistemas hidrossanitários prediais. PARC Pesquisa em Arquitetura e Construção, Campinas, SP, v. 7, n. 2, p. 102-113, 2016. DOI: https://doi.org/10.20396/parc.v7i2.8645049.

LIMA, Camila Fonseca Melo. Gestão do processo de projeto hidrossanitário. 2016. Dissertação (Mestrado em Construção Civil) - Escola de Engenharia, Universidade Federal de Minas Gerais, Belo Horizonte, 2016.

LOHMANN, Alberto. Avaliação de assentamentos e habitações permanentes construídos após desastres naturais de 2008 no Vale do Itajaí, SC. 2015. Tese (Doutorado em Arquitetura e Urbanismo). Programa de Pós Graduação em Arquitetura e Urbanismo, Universidade Federal de Santa Catarina, Florianópolis, 2015.

LORENZI, L. S.; SILVA FILHO, L. C. P. Impacto da aplicação da ABNT NBR 15575 para os projetos de edificações. In: ENCONTRO NACIONAL DE TECNOLOGIA DO AMBIENTE CONSTRUÍDO, 15., 2014, Maceió. Anais [...]. Porto Alegre: ANTAC 2014.

LORENZI, Luciani Somensi. Análise crítica e proposições de avanço nas metodologias de ensaios experimentais de desempenho à luz da ABNT NBR 15575 (2013) para edificações habitacionais de interesse sociais térreas.2013. Tese (Doutorado em Engenharia)- Programa de Pós-Graduação em Engenharia Civil, Universidade Federal do Rio Grande do Sul, Porto Alegre, 2013.

MAHL, R. L.; ANDRADE, J. J. O. Aplicabilidade da norma NBR 15.575/2008 edifícios habitacionais de até cinco pavimentos - desempenho: estudo de caso e análise crítica. In: ENCONTRO NACIONAL DE TECNOLOGIA DO AMBIENTE CONSTRUÍDO, 13., 2010, Canela. Anais [...]. Porto Alegre: ANTAC 2010.

MELO, E. A.; ELIAS, P. C. S.; FENATO, C. S. da P. M. Critérios básicos da norma de desempenho ABNT NBR 15.575/2013 aplicados a casa Botucatu/FGMF arquitetos. Akrópolis, Umuarama, v. 24, n. 2, p. 163-174, jul./dez. 2016. DOI: https://doi.org/10.25110/akropolis.v24i2.6335

MIRANDA, Singoala dos Santos. A influência da NBR 15575 na prática da arquitetura na cidade de Pelotas, RS. 2014. Dissertação (Mestrado em Arquitetura e Urbanismo) - Programa de Pós-Graduação em Arquitetura e Urbanismo da Universidade Federal de Pelotas, Pelotas, 2014.

MIYAZATO, Tarsila. Integração do sistema de aquecimento solar (SAS) ao projeto de edificações residenciais. 2012. Dissertação (Mestrado em Arquitetura e Urbanismo) - Faculdade de Arquitetura e Urbanismo, Universidade de São Paulo, São Paulo, 2012. 
MOURA, Rafael De Sousa Leal Martins. Catalogação de inovações tecnológicas na construção civil. 2015. Dissertação (Mestrado em Engenharia Civil) - Programa de Pós-graduação em Engenharia Civil, Universidade Federal do Ceará, Fortaleza, 2015.

NICOLINE, Elda. A norma de desempenho como orientação para a elaboração do código de obras de Santana Do Livramento - RS. 2015. Dissertação (Mestrado em Engenharia Civil) - Programa de Pós-Graduação em Engenharia Civil, Universidade de Santa Maria, Santa Maria, 2015.

OKAMOTO, Patricia Seiko. Os impactos da norma brasileira de desempenho sobre o processo de projeto de edificações residenciais. 2015. Dissertação (Mestrado em Ciências) - Escola Politécnica, Universidade de São Paulo, São Paulo, 2015

OLIVEIRA, V. M.; HIPPERT, M. A. S.; PERUGINI, M. M.; LIMA, J. Normas brasileiras contidas na norma de desempenho NBR 15575:20131. In: SIMPÓSIO BRASILEIRO DE QUALIDADE DO PROJETO NO AMBIENTE CONSTRUÍDO. 3.; ENCONTRO DE TECNOLOGIA DE INFORMAÇÃO COMUNICAÇÃO NA CONSTRUÇÃO. 6., 2013, Campinas. Anais [...]. Porto Alegre: ANTAC, 2013, p. 1579-1590.

OTERO, J. A.; SPOSTO, R. M. Modelo de análise de riscos baseada em matemática fuzzy para suporte à gestão do desempenho de edificações habitacionais. In: ENCONTRO NACIONAL DE TECNOLOGIA DO AMBIENTE CONSTRUÍDO, 16., 2016, São Paulo. Anais [...]. Porto Alegre: ANTAC, 2016, p. 3963-3976.

OTERO, J.A.; SPOSTO, R. M. Implantação da ABNT NBR 15575:2013 em empresas incorporadoras e construtoras a partir de processos de sistemas de gestão da qualidade. In: ENCONTRO NACIONAL DE TECNOLOGIA DO AMBIENTE CONSTRUÍDO, 15., 2014, Maceió. Anais [...]. Porto Alegre: ANTAC 2014.

OTERO, J.A.; SPOSTO, R. M. Caracterização da atuação de construtoras e incorporadoras de Goiânia-GO frente às normas de desempenho ABNT NBR 15575:2013. In: ENCONTRO NACIONAL DE TECNOLOGIA DO AMBIENTE CONSTRUÍDO, 16., 2016, São Paulo. Anais [...]. Porto Alegre: ANTAC, 2016, p. 3948-3962.

PAGLIARI, C. S.; COSTELLA, M. F.; PILZ, S. E. Especificação da vida útil dos sistemas construtivos a partir da NBR 15575, segundo a abordagem de projetos. PARC Pesquisa em Arquitetura e Construção, Campinas, SP, v. 9, n. 1, p. 47-56, 2018. DOI: https://doi.org/10.20396/parc.vgi1.8648828.

PINHEIRO, Gabriela Becker Abras. Contribuição ao estudo do processo de projeto de sistemas prediais. 2017. Dissertação (Mestrado em Construção Civil) - Escola de Engenharia, Universidade Federal de Minas Gerais, Belo Horizonte, 2017.

PISANI, M. A. J.; ZEIN, L. V. Habitação Social Brasileira no século XXI: quantidade x qualidade. In: ENCONTRO DA ASSOCIAÇÃO NACIONAL DE PESQUISA E PÓS-GRADUAÇÃO EM ARQUITETURA E URBANISMO, 3., 2014 , São Paulo. Anais [...]. São Paulo: ANPARQ, 2014.

PRADO, Ricardo Rodrigues. Análise da percepção dos moradores quanto ao desempenho de unidades de habitação de interesse social com base na norma de desempenho ABNT NBR 15575. 2018. Dissertação (Mestrado em Engenharia Civil) - Programa de Pós-Graduação em Engenharia Civil, Universidade Federal de Sergipe, São Cristóvão, 2018.

SANTOS, D. G.; CARVALHO, Pa. M.; CARVALHO, E. M.; FERREIRA, L. I. M.; VIANA, M. R. Desempenho de edificações residenciais: projetistas e empresas construtoras. In: ENCONTRO NACIONAL DE TECNOLOGIA DO AMBIENTE CONSTRUÍDO, 16., 2016, São Paulo. Anais [...]. Porto Alegre: ANTAC, 2016, p. 3299-3310.

SANTOS, D. G.; LINO, R. T.; SORAGGI, M. V. DE F.; OLIVEIRA, M. B. DE. A inclusão na região noroeste fluminense da NBR 15575 - Edificações habitacionais: Desempenho. Revista Interdisciplinar do Pensamento Científico, Itaperuna, v.1, n.3, p.138-150, jan./jun. 2017.

SANTOS, F.; HIPPERT, M. A. O impacto da norma de desempenho no processo de projeto. In: CONGRESSO NACIONAL DE EXCELÊNCIA EM GESTÃO, 12.; INOVARSE, 3., 2016, Rio de Janeiro. Anais [...]. Rio de Janeiro: INOVARSE, 2016. 
SANTOS, Flávia Maria Ávila dos. Impactos da aplicação da ABNT NBR 15.575/2013 nas empresas de edificações. 2017. Dissertação (Mestrado em Ambiente Construido) - Programa de Pós-Graduação em Ambiente Construído, Universidade Federal de Juiz de Fora, Juiz de Fora, 2017.

SANTOS, Paulo Ricardo Ramos. Investigação da adaptação organizacional de construtoras de Aracaju à implantação da norma de desempenho. 2018. Dissertação (Mestrado em Engenharia Civil) - Programa de PósGraduação em Engenharia Civil, Universidade Federal de Sergipe, São Cristovão, 2018.

SARVEZUK, L. C.; SILVA, J. R. R. da. Adaptações nas metodologias projetuais das empresas de construção civil de maringá oriundas da implementação da nova norma de desempenho de edificações habitacionais - NBR 15575/2013. In: ENCONTRO INTERNACIONAL DE PRODUÇÃO CIENTÍFICA UNICESUMAR, 9., 2015, Maringá. Anais [...]. Maringá: UNICESUMAR, 2015.

SILVA JR., Mauro Augusto. Parâmetros de desempenho incorporados em projetos de arquitetura com o uso de aplicativo de modelagem BIM. 2016. Dissertação (Mestrado em Habitação: Planejamento e Tecnologia) - Instituto de Pesquisas Tecnológicas do Estado de São Paulo, São Paulo, 2016.

SILVA, A. T.; KERN, A. P.; PICCOLI, R.; GONZÁLEZ, M. A. S. Novas exigências decorrentes de programas de certificação ambiental de predios e de normas de desempenho na construção. Arquitetura Revista, São Leopoldo, v.10, n. 2, p. 105-114, jul./dez. 2014. DOI: https://doi.org/10.4013/arq.2014.102.06

SILVA, F. A.; ARANTES, E. Verificação automática de requisitos de projetos da norma de desempenho nbr 15.575 a partir da adequação de regras da plataforma BIM Solibri Model Checker. In: SIMPÓSIO BRASILEIRO DE TECNOLOGIA DA INFORMAÇÃO E COMUNICAÇÃO NA CONSTRUÇÃO 1.; SIMPÓSIO BRASILEIRO DE GESTÃO E ECONOMIA DA CONSTRUÇÃO 10., 2017, Ceará. Anais [...]. Porto Alegre: ANTAC, 2017.

SILVA, Flávio Paulino de Andrade e. Verificação automática dos requisitos de projetos da norma de desempenho pela plataforma BIM Solibri Model Checker. 2017. Dissertação (Mestrado em Construção Civil). Escola de Engenharia, Universidade Federal de Minas Gerais, Belo Horizonte, 2017.

SOUZA, J. L. P.; KERN, A. P.; TUTIKIAN, B. F. Análise quantiqualitativa da norma de desempenho (NBR $\mathrm{n}^{\circ}$ 15.575/2013) e principais desafios da implantação do nível superior em edificação residencial de multipavimentos. Gestão e Tecnologia de Projetos, São Carlos, v. 13, n. 1, p. 127-144, 2018. DOI: https://doi.org/10.11606/gtp.v13i1.133842

SOUZA, Jonas Leonardo Pessanha de. Desafios na implantação do nível superior da Norma de Desempenho em edificação residencial em Novo Hamburgo/RS. 2016. Dissertação (Mestrado em Engenharia Civil) - Programa de Pós-Graduação em Engenharia Civil, Universidade do Vale do Rio dos Sinos, São Leopoldo, 2016.

\section{${ }^{1}$ Vitor Dias Lopes Nunes}

Engenheiro Civil. Mestre em Ambiente Construído pela Universidade Federal de Juiz de Fora. Endereço postal: Rua José Lourenço Kelmer, s/n - Campus Universitário Bairro São Pedro Juiz de Fora, MG, Brasil - 36036-900

\section{${ }^{2}$ Maria Aparecida Steinherz Hippert}

Engenheira Civil. Doutora em Engenharia de Produção. Professora na Universidade Federal de Juiz de Fora. Endereço postal: Rua José Lourenço Kelmer, s/n - Campus Universitário Bairro São Pedro Juiz de Fora, MG, Brasil - 36036-900

\footnotetext{
${ }^{3}$ Aldo Ribeiro de Carvalho

Estudante de Engenharia Civil na Universidade Federal de Juiz de Fora. Endereço postal: Rua José Lourenço Kelmer, s/n Campus Universitário Bairro São Pedro Juiz de Fora, MG, Brasil - 36036-900

\section{${ }^{4}$ Diana Fiori Rubim}

Estudante de Engenharia Civil na Universidade Federal de Juiz de Fora. Endereço postal: Rua José Lourenço Kelmer, s/n Campus Universitário Bairro São Pedro Juiz de Fora, MG, Brasil - 36036-900
} 